\title{
Welchen Respekt schulden wir dem Embryo?
}

\section{Die embryonale Stammzellforschung in medizinethischer Perspektive}

\author{
G. Maio
}

\section{Zentrum für Ethik und Recht in der Medizin, Universitätsklinikum Freiburg}

Verfolgt man die öffentliche Diskussion um die embryonale Stammzellforschung, so könnte man den Eindruck gewinnen, als ginge es hier um eine reine Pro-Kontra-Argumentation. Die Stammzelldebatte ist in unserem Land wie auch in anderen europäischen Ländern durch diese Neigung zur Polarisierung gekennzeichnet $(5,6)$, und es ist unter anderem Aufgabe der Ethik, zu verdeutlichen, dass die Komplexität des Problems nicht in dieser Dichotomisierung von Für und Wider aufgehen kann. Was kann die Ethik - und speziell die medizinische Ethik - zur Diskussion um die embryonalen Stammzellforschung konkret leisten? Aufgabe der Ethik ist es nicht, eine moralische Aussage über "gut" oder "schlecht" zu fällen; sie versteht sich vielmehr auf die Begründung der moralischen Aussage; sie muss daher die Vorverständnisse kritisch überprüfen, die in einem bestimmten moralischen Urteil mit transportiert werden. Speziell die medizinische Ethik kann für eine solche Fragestellung eine hilfreiche Disziplin sein, weil sie über das Instrumentarium der Moralphilosophie verfügt und im Idealfall gleichzeitig in der Lage ist, das Selbstverständnis und die praktischen Handlungssituationen der Medizin mit zu reflektieren.

Zwar wird gegenwärtig der Eindruck erweckt, als sei der etwaige Import von Stammzelllinien die zu lösende Kernfrage, die Aktualität korreliert hier jedoch nicht mit der Relevanz der Fragestellung. Denn selbst wenn die Problematik des Imports geregelt wäre, so bliebe uns immer noch das Kernproblem, das sich letztlich auch hinter der Importfrage verbirgt: die Frage nach der Schutzwürdigkeit des Embryos. Doch wie will man klären, wie schutzwürdig ein Embryo ist? Wie bei jeder ethischen Reflexion müssen auch hier drei Ebenen unterschieden werden, die zunächst kurz dargelegt werden sollen, um in einem zweiten Schritt mögliche Rechtfertigungmöglichkeiten der embryonalen Stammzellforschung zu prüfen.

\section{Drei Ebenen ethischer Reflexion}

\section{a. Ebene der Bewertungsbegründung}

Die abstrakteste Ebene der ethischen Reflexion ist die Ebene der Moralbegründung. Wie können wir nun begründen, dass ein Embryo zu schützen ist oder nicht zu schützen ist? Hier gibt es im Grunde zwei Zugangsmöglichkeiten: Nach der ideengeleiteten Begründung geht man davon aus, dass ein Embryo deswegen zu schützen ist, weil er ein Selbstzweck ist und aus sich heraus etwas Wertvolles darstellt. Nach dieser Begründungsform wird der Wert des Embryos ontologisch, also von seinem Sein her begründet. Nach der empiriegeleiteten Begründung geht man davon aus, dass ein Embryo deswegen zu schützen ist, weil er bestimmte nachweisbare Fähigkeiten besitzt. Hier ist die Begründung für die Schutzwürdigkeit also nicht mehr das Sein als solches, sondern ein bestimmtes Können. Beide Zugangsmög-

Dtsch Med Wochenschr 2002; 127: 160-163

(c) Georg Thieme Verlag Stuttgart · New York lichkeiten haben Vorzüge und auch Nachteile. Wer ideengeleitet argumentiert, setzt eine Idee voraus; in unserem Fall also die Idee, dass das Sein eines Embryos ein wertvolles Sein ist. Diese Idee kann aber nur postuliert, nicht jedoch bewiesen werden. Sie ist daher eine Glaubensaussage.

Dies nehmen die Vertreter der empiriegeleiteten Denkrichtung zum Anlass für Kritik und sagen daher, dass es nicht allein das Sein, sondern nur das mit bestimmtem Können verbundene Sein ist, das den Embryo schützenswert macht. Doch auch die Vertreter der empiriegeleiteten Denkrichtung bleiben am Ende den Beweis schuldig, denn sie können zwar sagen, der Embryo besitze eine bestimmte Fähigkeit oder besitze sie nicht, aber sie können nicht festlegen, dass aus dieser empirischen Tatsache normativ etwas abzuleiten ist. Fakten sagen für sich nichts über die Bedeutung dieser Fakten aus, die "Bedeutung" ergibt sich eben erst aus der "Deutung", aus der Interpretation der Fakten. Allein dass wir z.B. empirisch nachweisen, dass ein Embryo stärker auf den mütterlichen Organismus angewiesen ist, als wir bisher dachten, sagt noch nichts darüber aus, ob wir deswegen den extrakorporalen Embryo weniger schützen sollen. Aber ein Urteil über den Embryo zu fällen und diese Tatsache dabei zu ignorieren, ist wiederum leichtfertig und gefährlich, weil damit Urteile möglicherweise auf dem Boden von falschen Vorstellungen gefällt werden können. Eine Idee, die nicht die empirischen Tatsachen zur Kenntnis nimmt, steht in Gefahr, sich als irrtümliche Idee zu erweisen. Daher ist es für eine sachgerechte moralische Einordnung des Embryos wichtig, sich für die naturwissenschaftlichen Erkenntnisse zu interessieren.

1. Schlussfolgerung: Sowohl die ideengeleitete wie die empiriegeleitete Begründungstheorie beruht auf Vorannahmen und nicht auf Beweisen. Je nachdem, welches Begründungsmuster man wählt, gelangt man zu unterschiedlichen Bewertungen der Schutzwürdigkeit des Embryos.

\section{b. Ebene der Bewertungskriterien}

Als Kriterien für die Bewertung werden insbesondere folgende Argumente diskutiert, ohne dass in diesem Rahmen näher darauf eingegangen werden kann. 1. Potenzialitätsargument: Dieses Argument besagt, dass wir den Embryo nicht für das was er ist schützen wollen, sondern für das, was aus ihm werden kann. Der Streit geht hier um die genaue Differenzierbarkeit von aktiver und passiver Potenzialität. 2. Identitätsargument: Dieses besagt, dass ein Embryo ab dem Moment zu schützen ist, da er die Identität eines Menschen besitzt. Hier geht es um die Frage der Konkretisierung dieser Identität, die nicht vom Individualitätsbegriff zu trennen ist. Woran möchte man Identität und Individualität festmachen, und liegt beim frühen Embryo eine Individualität vor, solange eine Mehrlingsbildung möglich ist? 3. Kontinuitätsargument: Es besagt, dass der Embryo deswegen ab dem Moment zu schützen ist, ab dem keine weiteren ethisch relevanten Zäsuren in seiner weiteren Entwicklung auszumachen sind. Der Streit geht hier darum, ob es noch andere moralisch relevante Zäsuren außer der Fertilisation gibt, wie 
z.B. die Nidation. 4. Gattungszugehörigkeitsargument: Nach diesem Argument ist der Embryo nicht etwa für die faktischen Fähigkeiten und Potenziale zu schützen, sondern für seine $\mathrm{Zu}-$ gehörigkeit zur Spezies Mensch.

\section{c. Ebene der moralischen Bewertung}

Auf der Stufe der moralischen Bewertung lassen sich im Wesentlichen zwei Bewertungsmodelle ausmachen (7). Das erste Bewertungsmodell würde ich "Personmodell" nennen. Damit meine ich die Bewertung, die davon ausgeht, dass der Embryo so behandelt werden soll wie eine Person. Hiernach wäre der Embryo ab dem Moment der Fertilisation genauso zu schützen wie ein Erwachsener. Auf der Grundlage dieser Schutzwürdigkeit wäre jegliche Forschung an Embryonen als illegitim zu erachten, weil es keinen auch noch so hohen Nutzen geben kann, der es rechtfertigen würde, die personalen Grundrechte des Embryos zu verletzen, es sei denn es stünde Leben gegen Leben (10). Das deutsche Embryonenschutzgesetz folgt in seiner Logik genau diesem Modell. Als zentrale Argumente dieses Modells fungieren das Potenzialitäts- und das Gattungszugehörigkeitsargument. Das zweite Bewertungsmodell möchte ich das »Objektmodell" nennen. Hiernach wird der Embryo als reine Sache, als "Zellhaufen» betrachtet, der keine eigenen Schutzrechte hat. Die Forschung an Embryonen wäre nach diesem Modell nur dann rechtfertigungsbedürftig, wenn außerembryonale Güter damit verletzt würden. Als Begründung hierfür wird insbesondere das Argument des "Wertungswiderspruchs" verwendet, das darauf hinweist, dass Embryonen in vivo weniger geschützt sind als in vitro. (2) Ist der Embryo ein Mensch oder eine Sache? Um die Entscheidung für eines dieser Bewertungsmodelle geht nun der Streit.

Doch die Möglichkeiten der Bewertung des Embryos erschöpfen sich bei kritischer Betrachtung nicht darin, ihn entweder als Person und damit als vollgültiges Mitglied der sittlichen Gemeinschaft oder als Sache zu betrachten und ihn damit gänzlich aus der Gemeinschaft des Menschen auszuklammern. Der Embryo kann auch in einer Weise betrachtet werden, dass er noch nicht vollgültiges Mitglied der Gemeinschaft und gleichzeitig aber auch nicht nur reines Objekt ist. Dies wäre Grundlage für ein drittes Bewertungsmodell des Embryos, das ich als Progredienzmodell oder auch "Respektmodell" bezeichnen möchte. Nach diesem Bewertungsmodell geht man davon aus, dass die Schutzwürdigkeit des Embryos in Abhängigkeit mit seiner Entwicklungsstufe stetig zunimmt. Ein früher Embryo wie in seinem Präimplantationsstadium wäre hiernach weniger schutzwürdig als ein Fetus im 5. Schwangerschaftsmonat $(1,2)$. Die Attraktivität dieses Modells ist aber weniger diese gradualistische Auffassung von Schutzwürdigkeit, sondern vielmehr die in diesem Modell enthaltene Prämisse, dass auch das früheste embryonale Leben Respekt verdient und nicht als frei verfügbares Material betrachtet werden kann. Gleichzeitig würde man mit dem Respektmodell die Gefahr vermeiden, dass dem Embryo ethische Konzepte »übergestülpt" werden, die eher auf moralfähige Personen zugeschnitten sind.

\section{Wann bringen wir dem Embryo Respekt entgegen?}

Wie können wir zum Ausdruck bringen, dass wir - obwohl wir Embryonen zu Forschungszwecken benutzen - diesen Embryonen Respekt entgegenbringen? Diese Frage lautet nichts anderes als: Wie können wir ausdrücken, dass wir diese Embryonen als etwas Wertvolles ansehen (12)? Geht das überhaupt? Zunächst einmal schließt die Manifestation von Respekt selbst das Töten des Embryos nicht grundsätzlich aus. Töten und Respekt können miteinander vereinbar sein, wenn der Akt des Tötens durch eine entsprechende Haltung begleitet wird. So kann man beispielsweise auch Tiere töten und dennoch Ihnen Respekt entgegenbringen (9). Daher wäre es zu kurzsichtig, wenn man schon die Forschung als solche als mit dem Respektbegriff nicht vereinbar ansähe. Für die Erfüllung des Respektes ist nicht nur die Handlung als solche relevant, sondern in besonderer Weise auch die Haltung, die hinter der Handlung steckt. Von der Haltung her würde es Ausdruck von Respekt sein, wenn man den Tod des Embryos bedauern würde. Man müsste den Embryo somit als etwas betrachten, das nicht einfach ersetzt werden kann, sondern als etwas, dessen Verlust uns nicht gleichgültig ist. (11) Von der Handlung her würde respektvoller Umgang bedeuten, dass man alles tut, um die Existenz des Embryos nicht leichtfertig aufs Spiel zu setzen. Wenn man den Tod z.B. nur in besonderen Konfliktfällen zuließe, wäre dies ein Ausdruck von Respekt. Die entscheidende Frage für die Manifestation von Respekt lautet also: Ab wann liegt eine solche besondere Konfliktsituation vor, dass wir sagen können, es ist dieser Konflikt, der uns die Verwendung von Embryonen aufnötigt und nicht der fehlende Respekt vor dem Embryo? Diese Frage ist nichts anderes als die Frage nach den hohen Gütern, die eine Verwendung von Embryonen für die Stammzellforschung rechtfertigen könnten.

2. Schlussfolgerung: Dem Embryo kann in der Weise Respekt entgegengebracht werden, dass wir seinen Tod nur in den Notsituationen hinnehmen, die durch einen gravierenden Konflikt gekennzeichnet sind.

\section{Die Hilfspflicht als Rechtfertigung der Embryonenforschung?}

Nun liegt es für viele Menschen auf der Hand, dass ein abzuwägendes Gut das der Hilfe für den Kranken ist. Diese Frage berührt den Kern der ärztlichen Identität, ist doch gerade die Konstellation von Not und Hilfe die zentrale Grundlage ärztlichen Tuns. Der Not des Patienten steht die Hilfe des Arztes gegenüber. Dem Arzt kann es also nicht gleichgültig sein, ob er helfen kann oder nicht. Gleichwohl gibt es zwei unterschiedliche Arten von Hilfe, für den Arzt wie für jeden Menschen. Es gibt die verbindliche Rechtspflicht mit unbedingter Sollensaufforderung und eine reine Tugendpflicht, die - wie z.B. die Pflicht zur Nächstenliebe - nicht erzwungen werden kann (8). Wenn also erwiesen werden könnte, dass die embryonale Stammzellforschung nicht nur als Tugendpflicht, sondern als eine verbindliche Rechtspflicht zu sehen wäre, um die Not der Kranken zu lindern, so wäre dies eine Konfliktsituation, die es rechtfertigen könnte, das Verwenden von Embryonen zu erwägen, ohne damit dem Respekt zuwiderzuhandeln. Damit für die Person X (also hier der Arzt) eine solche verbindliche Hilfspflicht gegenüber der Person Y (hier der Patient) besteht, müssten aus ethischer Sicht (2) mindestens vier Bedingungen erfüllt sein: 1. Bedingung: Das Leben, die Gesundheit oder andere relevante Interessen von Y müssen gefährdet sein. 2. Bedingung: Die Handlung von X muss allein oder in Verbindung mit anderen Handlungen notwendig sein, um diesen Verlust relevanter Interessen zu verhindern. 3. Bedingung: Die Handlung von X muss allein oder in Verbindung mit anderen Handlungen fähig sein, den Verlust zu verhindern. 4. Bedingung: Die Hilfe, die Y erwarten kann, muss im Vergleich zu den Gefahren, die durch die Handlung $\mathrm{X}$ in Kauf genommen werden, überwiegen.

Diese vier Grundvoraussetzungen für die Postulierung einer Notsituation mit entsprechender Hilfsverpflichtung machen 
deutlich, daß einige Fragen noch zu klären sind, bevor eine solche Rechtspflicht zur Stammzellenforschung auch tatsächlich formuliert werden kann. Aufschlussreich ist die 2. Bedingung, die auf die Frage abhebt: Wie notwendig ist Stammzellforschung für das angestrebte Ziel der Hilfe? Es ist gegenwärtig noch offen, ob die embryonale Stammzellforschung nicht vielleicht doch entbehrlich sein könnte. Eine Notlage, in der die embryonale Stammzellforschung tatsächlich die alternativlose Methode darstellte, hätte man aber erst ab dem Moment, da klar wäre, dass die adulte Stammzellforschung die Ergebnisse nicht herbeiführen kann. Wenn dies einmal klar werden sollte, dann stünden wir allerdings vor der Situation, dass wir die Hilfe für Kranke mit einer Latenz von einigen Jahren erzielt hätten, obwohl wir sie hätten früher erzielen können. Man hätte also, so könnte man argumentieren, die Hilfe für die Kranken einige Jahre lang dem Respekt vor dem Embryo untergeordnet. Kann man aber so tatsächlich argumentieren?

Hierauf gibt uns die 3. Bedingung für die Hilfspflicht Antwort, die darauf abhebt, dass von einer Verpflichtung zur Forschung nur dann gesprochen werden kann, wenn diese Forschung tatsächlich eine Hilfe bedeutet. Wenn man nun diese Voraussetzung für erfüllt erklärte und behauptete, dass die Forschung an embryonalen Stammzellen fähig sei, Patienten zu helfen, so erläge man einem logischen Argumentationsfehler. Denn wenn gesichert wäre, dass die Forschung Hilfe brächte, so bedürfte es der Forschung nicht, sondern lediglich der Anwendung. Es ist gerade konstitutives Merkmal der Forschung, Hypothesen zu überprüfen, und die Unsicherheit des Ergebnisses ist der Forschung inhärent. Die Gleichsetzung von wissenschaftlicher Erkenntnis und Nutzen für den Patienten erscheint in dieser Form nicht haltbar (8), und daher ist diese dritte Bedingung zum momentanen Zeitpunkt nicht erfüllt.

Aber es lässt sich hier einwenden, dass letztlich auch bei jeder Standardtherapie diese Sicherheit nie gegeben ist, und es lässt sich auch einwenden, dass der Verzicht auf die Forschung uns die Möglichkeit nimmt, herauszufinden, ob sie nicht vielleicht doch Hilfe bedeuten könnte. Daher kann die Forschung für den Hilfsaspekt keineswegs als unerheblich betrachtet werden. Aber es wäre korrekter, von potenzieller Hilfe zu sprechen und vor allem von einer Hilfe, die erst zukünftigen Patienten zugute käme, anstatt eine faktische und aktuelle Hilfe zu suggerieren, die nicht vorliegt.

Weil die Hilfe nur potenziell und prospektiv und überdies auch nicht alternativlos vorliegt, erscheint der Hinweis auf eine verbindliche Verpflichtung mit unbedingter Sollensaufforderung kaum begründbar. Würde man schon die potenzielle Hilfe zur unbedingten Rechtspflicht machen, so käme man in Begründungsnot, warum man andere potenzielle Hilfen nicht zur Pflicht macht.

3. Schlussfolgerung: Wenn man davon ausgeht, dass die Verwendung von Embryonen für die Stammzellforschung nur dann mit dem Respekt vereinbar ist, wenn ein Konflikt mit einer verbindlichen Hilfspflicht vorliegt, dann wäre es unter den momentanen Bedingungen kein Ausdruck von Respekt, wenn Embryonen für die Stammzellforschung benutzt würden.

\section{Die Forschungsfreiheit als Rechtfertigung der Embryonenforschung?}

Nun stellt sich aber die Frage, ob man die Güterkollision tatsächlich nur an einer etwaigen Hilfspflicht festmachen kann. Bei genauer Betrachtung gibt es noch ein weiteres hohes Gut, das eine Konfliktsituation begründen könnte, und das ist das
Gut der Forschungsfreiheit. Die Argumentation würde also lauten: Wir tun alles, um Embryonen nicht leichtfertig aufs Spiel zu setzen, aber im Falle der Stammzellforschung liegt ein schwerwiegender Konflikt mit der Forschungsfreiheit als hochstehendem Gut vor, so dass das Handeln in dieser Konfliktlage nicht als Mangel an Respekt gedeutet werden kann. Dies ist letztlich die Argumentation der Mehrheit des nationalen Ethikrates. In der Tat ist die Forschungsfreiheit von der deutschen Verfassung her als sehr hohes Gut veranschlagt, und in der Tat muss daher auch die Einschränkung der Forschungsfreiheit gut begründet werden. Gleichwohl gilt natürlich auch die Forschungsfreiheit nicht absolut, denn sonst wäre ja gerade die Institution der Ethikkommission widersinnig. Über die Einschränkung der Forschungsfreiheit in Bezug auf die Forschung mit Embryonen ist schon in den 80er Jahren diskutiert worden. In Deutschland kam man zu der politischen Entscheidung, dass die Schutzpflichten dem Embryo gegenüber stärker zu gewichten seien als das hohe Gut der Forschungsfreiheit. Selbst die Länder, die die Embryonenforschung zugelassen haben, ließen diese nur für die Forschungsziele im Bereich der Reproduktionsmedizin zu, also für den Bereich, der in direkter Beziehung zum Embryo steht. Die Argumentation lautete: Die jetzige Forschung an Embryonen wird zugelassen, damit zukünftige Embryonen davon profitieren können. Im Vordergrund stand also der gruppenspezifische potenzielle Nutzen. Bei der jetzt anstehenden embryonalen Stammzellforschung besteht nun keine Verbindung zum Wohl zukünftiger Embryonen. Das heißt, wir würden heute Embryonen de facto im Interesse Dritter verwenden, und eine solche fremdnützige Forschung wurde in den 80er Jahren von den allermeisten Ländern für nicht vertretbar gehalten. Wenn nun heute die Forschungsfreiheit als das ausschlaggebende Argument für die fremdnützige Embryonenforschung gelten soll, so bleibt die Frage unbeantwortet, warum denn diese Forschungsfreiheit in den 80er Jahren beispielsweise der britischen Regierung nicht ausgereicht hat, um speziell die fremdnützige Forschung an Embryonen zu rechtfertigen. Erst recht stellt sich diese Frage für die Bundesrepublik, denn wenn die Forschungsfreiheit - wie jetzt argumentiert wird den Schutzpflichten für den Embryo überlegen sei, dann hätte dies auch schon 1990 so sein müssen. Begründen könnte man eine solche unterschiedliche Bewertung nun auf dreierlei Weise. 1. Man könnte sagen, dass die Forschungsfreiheit heute einen höheren Stellenwert hat als 1990, was freilich keinen Sinn macht. 2. Man könnte auch sagen, die Rahmenbedingungen hätten sich seitdem geändert, und es habe sich durch das damals ungeahnte Potenzial der Embryonenforschung eine neue Situation ergeben, die zu neuen Bewertungen Anlass gebe. Diese Begründung rekurriert allerdings nicht auf die Forschungsfreiheit, sondern vielmehr auf den therapeutischen Nutzen. 3. Schließlich könnte man auch sagen, dass sich das Schutzniveau des Embryos geändert habe, weil die gesellschaftliche Akzeptanz des Personmodells heute weniger vorhanden ist als vor 10 Jahren. Dieses Argument macht aber die Schutzwürdigkeit des Embryos letztlich davon abhängig, für wie groß das Opfer angesehen wird, das mit dem zu gewährenden Schutz verknüpft ist. Wenn wir also so argumentieren wollen, dass uns der Preis des Schutzes zu hoch geworden ist, so müsste man dies auch so benennen. Der Hinweis auf die Forschungsfreiheit jedenfalls kann hier nicht weiterhelfen.

4. Schlussfolgerung: Auch das Argument der Forschungsfreiheit kann nicht ausreichen, um eine solche Konfliktsituation vorauszusetzen, aus der heraus die Forschung an Embryonen als eine respektvolle Behandlung gesehen werden könnte. 


\section{Der Zustand des Verwaistseins des Embryos als Argument für die Forschung?}

Es bleibt aber noch eine weitere Möglichkeit, zu begründen, warum eine etwaige Verwendung von Embryonen doch mit dem Respekt vereinbar sein könnte. Diese bezieht sich nicht auf eine etwaige Konfliktsituation, sondern auf die spezifische Lage des verwaisten Embryos. So könnte man argumentieren: wir drücken Respekt dadurch aus, dass wir alles tun, um den Tod des Embryos zu verhindern. Dies bedeutet, dass für den Fall, dass der Embryo eine Chance hat, sich zu einem Menschen zu entwickeln, ihm diese Chance unter keinen Umständen verwehrt wird. Jeder Embryo, der Aussicht auf Menschwerdung hat, wird geschützt und sein Tod verhindert. Damit Embryonen nicht deswegen sterben müssen, weil sie nach einer künstlichen Befruchtung möglicherweise verwaisen, wird auch jede Anstrengung unternommen, um auch den Tod verwaister Embryonen zu verhindern. Wenn aber nun trotz all dieser Anstrengungen schicksalhafterweise immer noch Embryonen übrigbleiben, deren Tod nicht mehr verhinderbar ist, so läge nach dieser Argumentation eine andere Situation vor, die einen anderen Gehalt von Respekt bedingen würde. In dieser ausweglosen Situation, da der Tod nicht mehr aufzuhalten ist, - so könnte man argumentieren - wäre es demnach mit dem Respekt vor dem Embryo vereinbar, dass man ihn zur Forschung verwendete, weil nun nur noch eine Güterabwägung zwischen Verwerfen und Forschen anstünde. Welchen Sinn macht es, - so fragen sich viele Menschen - die Forschung an Embryonen für unmoralisch zu erachten, wenn die Alternative zur Forschung nur noch das Verwerfen ist?

\section{Kernfrage: Wodurch ist der Respekt vor dem Embryo begründet?}

Die entscheidende Frage am Ende lautet daher: Was ist es denn genau, was wir respektieren wollen. Wie wollen wir die Notwendigkeit des Respektes begründen? Oder konkret ausgedrückt: Was wollen wir da eigentlich schützen, wenn wir einen Embryo schützen, bevor er verworfen werden muss? In jedem Fall schützen wir da keinen zukünftigen Menschen, weil aus diesem Embryo ein Mensch nie werden kann. Wenn wir aber nun sagen, dass die verwaisten Embryonen - ihrer Zukunftsaussichten wegen oder äußerer Bedingungen wegen - weniger schutzwürdig sind als die nicht verwaisten, so fällen wir damit eine Grundentscheidung. Wir entscheiden uns nämlich dafür, den Wert des Embryos von seinen Fähigkeiten, von seinem Umfeld und von seiner Zweckbestimmung durch den Menschen abhängig zu machen. Gleichzeitig entscheiden wir uns dagegen, den Embryo als Embryo mit einem nur in seinem Sein begründeten Wert zu sehen. Denn von seinem Sein her besteht ja zwischen dem verwaisten und dem nicht verwaisten Embryo kein Unterschied. Stellen wir uns eine Petrischale mit zwei Embryonen vor, ein Embryo, der von den Eltern nicht mehr gebraucht wird, weil diese doch keine Schwangerschaft mehr wünschen und daneben ein anderer Embryo, der am nächsten Tag transferiert werden soll. Wenn man nun einen moralisch relevanten Unterschied macht zwischen diesen beiden Forschungshandlungen, so hat man in dieser Beurteilung bereits die Grundentscheidung getroffen, dass nicht die Existenz des Embryos als solche, sondern nur seine Situation für die Schutzwürdigkeit von Belang wäre. Der moralische Status des Embryos wäre nach dieser Argumentation daher nicht ontologisch festzumachen, sondern er wäre von seinen faktischen Fähigkeiten und von äusseren Bedingungen abhängig.
6. Schlussfolgerung: Die grundsätzliche Frage, die die gesamte Diskussion um die Stammzellforschung aufwirft, besteht also darin, ob wir dem Embryo allein aus seiner bloßen Existenz heraus Schutzwürdigkeit zusprechen wollen oder ob das Ausmaß der Schutzwürdigkeit von Faktoren abhängen soll, die außerhalb seiner selbst liegen. Und so führt uns dieses Ergebnis zur Ausgangsüberlegung zurück, denn die Beantwortung dieser Frage hängt davon ab, ob wir für die moralische Bewertung unseres Handelns eher der ideengeleiteten Begründung anschließen oder eher empiriegeleitet argumentieren wollen. Das Resultat des Urteils hängt am Ende von der Wahl unseres Begründungsmusters ab. Moralische Urteile hängen vom gewählten Denkmodell ab, also von den Vorannahmen, die vor dem Urteil bereits akzeptiert wurden. Die Frage des Umgangs mit Embryonen kann nicht als eine Frage nach der einen Wahrheit betrachtet werden. Auf der Grundlage der unterschiedlichen Begründungsmuster stehen sich auch unterschiedliche "Wahrheiten" gegenüber. Daher ist es für die Diskussion förderlich, wenn man anstatt Scheinargumente zu benutzen, sich offen zu der einen oder anderen Wahrheit bekennt. Gleichsam ist es wichtig, darüber nachzudenken, auf welche Inhalte sich eine Gesellschaft verständigen kann, auch wenn sie über kein einheitliches konsensfähiges Begründungsmodell verfügt. Worin liegt die gemeinsame Basis, die Schnittmenge, die von beiden Wahrheitszugängen akzeptiert werden kann? Mit dem Begriff des Respektes mag eine solche denkbare Lösung aufgezeigt sein.

\section{Literatur}

1 Bayertz K. Drei Thesen zum moralischen Status menschlicher Embryonen in vitro. Ethisch-philosophische Aspekte. In: Fortpflanzungsmedizin in Deutschland. Baden-Baden: Nomos, 2001, S. 81-84

2 Beauchamp T. L., Principles of biomedical ethics. - 5. ed.. - New York : Oxford Univ. Press, 2001

3 Birnbacher D. Embryonenschutz in Gefahr? Universitas 2000; 55: 409-419

4 Kopelman L. Respect and the retarded. In: Kopelman L., Moskop JC, eds. Ethics and mental retardation. Reidel, 1984, S.65-85

5 Maio G. Zum Für und Wider der embryonalen Stammzellforschung. Forum-Deutsche Krebsgesellschaft 2001; 16: 8-12

6 Maio G. Die ethische Diskussion um embryonale Stammzellen aus internationaler Sicht - das Beispiel Italien. Zeitschr Med Ethik 2001; 47: 299-309

7 Maio G. Die Präimplantationsdiagnostik als Streitpunkt. Welche $\mathrm{Ar}$ gumente sind stichhaltig und welche nicht? Dtsch Med Wochenschr 2001; 126: 889-895

8 Maio G. Ethik der Forschung am Menschen. Philosophische Analyse und historischer Kontext. (Medizin und Philosophie, Bd. 6) Stuttgart: Frommann-Holzboog, 2002

9 Meyer M.J., Nelson LJ. Respecting what we destroy. Reflections on human embryo research. Hastings Center Report 2001; 31,1: 16-23

10 Schockenhoff E. Die Ethik des Heilens und die Menschenwürde. Moralische Argumente für und wider die embryonale Stammzellforschung. Zeitschr Med Ethik 2001; 47: 235-257

11 Steinbock B. The moral status of extracorporeal embryos. Pre-born children, property or something else. In: Dyson A., Harris J, eds. Ethics and biotechnology. London New York: Routledge, 1997, S. 79-92

12 Wolf U. Das Problem des moralischen Sollens. Berlin: de Gruyter, 1984, p. 189

PD Dr. G. Maio

Zentrum für Ethik und Recht in der Medizin

Universitätsklinikum Freiburg

Elsässer Straße 2m, Haus 1a

79110 Freiburg

Tel.: 0761-270-7267

Fax.: 0761-270-7268

E-Mail: maio@sfa.ukl.uni-freiburg.de 